\title{
Analysis and Design of Control Dynamics of Manipulator Robot's Joint Drive
}

\author{
Bukhar Kussainov \\ Institute of Control Systems and Information Technologies, Almaty University of Power Engineering and Telecommunications, \\ Almaty 050013, Kazakhstan
}

\begin{abstract}
A system of automatic control (SAC) of the electric drive of rotary link of manipulator robot (MR) is considered. The variables of mechanical load of drive, depending of changes of a spatial configuration of MR in the course of motion, mass and dimensions of the moved payloads, etc., are received from the motion equation of rotary link of MR. Changes of mechanical load can be unknown and cause the essential deterioration in dynamical properties (speed, damping, etc.) of the SAC of drive. With the aim to stabilize the desired dynamical properties of SAC the algorithm of drive control is proposed which is adaptive to changes of mechanical load. The unknown parameters of drive load, necessary to form the adaptive control algorithm, are identified via observing device (OD). The work algorithm of OD to identify the unknown parameters of drive load is proposed. For the proposed control and work of OD algorithms the block diagram of drive control is construction. The simulation results on computer proved that in considered SAC of drive the stabilization of desired dynamical properties ensured.
\end{abstract}

Key words: Manipulator robot, control dynamics of joint drive, load adaptive control system, observing device.

\section{Introduction}

Dynamic equations of the multi-joint manipulator robot (MR) are the set of motion equations of its separate mobility degrees being interdependent on the motion coordinates of mobility degrees and, therefore, being the non-linear and non-stationary differential equations [1-5]. For an illustration of interference of mobility degrees at each other it is sufficient to consider the motion equations of any two next links of MR making two rotary or one rotary and one translational motions in one plane [2]. In the equation of rotary degree of mobility of MR it is possible to allocate a moment of inertia of joint and terms caused by inertial forces from interference of movements and gravity of links of MR. These moments of inertia of joint and additional terms of the motion equation of a link of MR are variables and depend on changes of a spatial configuration of MR in the course of motion,

Corresponding author: Bukhar Kussainov, candidate of technical sciences, senior lecturer, research fields: dynamics and control of technical systems with uncertain parameters. mass and dimensions of the moved payloads and also technology factors, etc. [3]. As each link of MR is set in motion by its drive, the motion equation of a link of MR should be added with the equations of dynamics of the drive $[2,4]$. The moment of inertia of joint and the equation terms of interference and gravity of links of MR in common with the corresponding moment of inertia and moment of mechanical resistance of the drive itself and specified to the rotating shaft of the drive motor become the moment of inertia and the moment of resistance of mechanical load of the link drive of MR [5].

Thus the joint drives of MR experience the variable mechanical loads characterized by changes of the moment of inertia and the moment of resistance of mechanical load. These mechanical values for the system of automatic control (SAC) of the drive have the following sense: the moment of inertia is the parameter (coefficient of the differential equation) and the moment of resistance (external moment) of load is the external disturbing signal of the SAC of drive. Changes of these variables of load can happen over a 
wide range and cause the essential deterioration in the dynamic properties (speed, damping, etc.) of the SAC of drive. There is a problem of creation in each joint of MR of a load adaptive SAC of drive in which dynamic properties do not depend on changes of load. Therefore, it is necessary to define and use the variable parameters of load of drive for the stabilization of desirable dynamic properties of the SAC of drive. If MR moves unknown payloads, then these variables of load will be not only changing, but they will be also unknown. Both the unknown and changing parameters of load are necessary to form a load adaptive control algorithm of SAC are identified (defined, estimated) by means of the observing devices (OD) [6].

\section{Preconditions for Analysis the Problem of Control of Motions of Manipulator Robot}

The dynamical equation of MR with $n$ mobility degrees [1, 2] is

$$
M(\vec{q}) \ddot{\vec{q}}+V(\vec{q}, \dot{\vec{q}})+G(\vec{q})=\vec{\tau}
$$

where $M(\vec{q})$-the $n \times n$ matrix of inertia coefficients; $V(\vec{q}, \dot{\vec{q}})$-the $n$-vector of Coriolis and centripetal components; $G(\vec{q})$-the $n$-vector of gravity; $\vec{\tau}$-the $n$-vector of generalized forces; $\vec{q}$-the $n$-vector of joint variables (generalized coordinates); $\dot{\vec{q}}, \ddot{\vec{q}}$-the $n$-vectors of joint velocities and accelerations.

For the feature detection of spatial motions of MR is sufficient to consider motions of two-link MRs on the plane. As in Ref. [2] let us consider a two-link revolute/prismatic MR (in polar coordinate system), at which the joint-variables, the joint-velocities and the corresponding generalized forces vectors are:

$$
\vec{q}=\left[\begin{array}{l}
\varphi \\
r
\end{array}\right], \dot{\vec{q}}=\left[\begin{array}{c}
\dot{\varphi} \\
\dot{r}
\end{array}\right], \quad \vec{\tau}=\left[\begin{array}{l}
n \\
f
\end{array}\right]
$$

where $\varphi, \dot{\varphi}$-the joint-variable and the joint-velocity of a revolute link; $r, \dot{r}$-the joint-variable and the joint-velocity of a prismatic link; $n$-the torque of a revolute link; $f$-the force of a prismatic link.
Let us enter designations: $m_{1}$ - the mass, $J_{1}$ - the moment of inertia concerning the revolution axis of the 1 (revolute) link; $m_{2}$-the mass, $J_{2 c}$-the central moment of inertia of the 2 (prismatic) link of MR; $r_{1}$-the distance from the end of the 2-link to its center of masses; $m_{p}$ - the mass, $J_{p c}$-the central moment of inertia of the next links or of the payload (for the last link) of MR.

In accordance with Eq. (1) the dynamical equations of two-link polar MR [4] are:

$$
\begin{gathered}
{\left[\begin{array}{cc}
J_{1}+m_{2}\left(r-r_{1}\right)^{2}+J_{2 c}+m_{p} r^{2}+J_{p c} & 0 \\
0 & m_{2}+m_{p}
\end{array}\right]} \\
\times\left[\begin{array}{c}
\ddot{\varphi} \\
\ddot{r}
\end{array}\right]+\left[\begin{array}{c}
2\left(m_{2}\left(r-r_{1}\right)^{2}+m_{p} r\right) \dot{\varphi} \dot{r} \\
\left(m_{2}\left(r-r_{1}\right)+m_{p} r\right) \dot{\varphi}^{2}
\end{array}\right] \\
+\left[\begin{array}{c}
\left(m_{2}\left(r-r_{1}\right)+m_{p} r\right) g \cos \varphi \\
\left(m_{2}+m_{p}\right) g \sin \varphi
\end{array}\right]=\left[\begin{array}{l}
n \\
f
\end{array}\right]
\end{gathered}
$$

We can see that motions on $\varphi$ and $r$ coordinates of two-link MR are interdependent and the dynamical Eq. (2) is the non-linear and non-stationary differential equations.

The equation of revolute movement on the $\varphi$ coordinate we will write as follows:

$$
J_{\varphi}\left(r, m_{p}\right) \ddot{\varphi}+M_{\varphi}\left(r, \varphi, m_{p}\right)=n
$$

where $\quad J_{\varphi}\left(r, m_{p}\right)=J_{1}+m_{2}\left(r-r_{1}\right)^{2}+J_{2 c}+$ $m_{p} r^{2}+J_{p c}$ is the moment of inertia of joint of MR; $M_{\varphi}\left(r, \varphi, m_{p}\right)=2\left(m_{2}\left(r-r_{1}\right)^{2}+m_{p} r\right) \dot{\varphi} \dot{r}$

$+\left(m_{2}\left(r-r_{1}\right)+m_{p} r\right) g \cos \varphi$ is the moment of interference and gravity of links of MR.

In relation to multi-link MR the quantities $J_{\varphi}$ and $M_{\varphi}$ of rotary link mean the following [3]: $J_{\varphi}=$ $J_{\varphi}(\vec{q}, \vec{\xi})$ is the moment of inertia of a joint of MR changing in dependence on a $\vec{q}$ vector of generalized coordinates and a $\vec{\xi}$ vector of parameters of MR and its payload (geometrical, weight-inertial parameters, etc.); $M_{\varphi}=M_{\varphi}(\vec{q}, \overrightarrow{\dot{q}}, \vec{\xi})$ is the resistance moment of a joint of MR and its changes are caused by mutual influence of movements on mobility degrees of MR, moments from the gravity of links and a payload of MR, etc. 


\section{Equations of a Revolute Link Drive with a Variable Mechanical Load}

Let us consider the equations of the drive of rotation of a link of MR being the plant (control object) and consisting of the amplifier of power, the DC electric motor and the mechanical transfer (reducer) with the variable mechanical load [5].

Let us write equations for the "electrical" part of the drive [5, 7]. The equation for an electric circuit of the DC motor is:

$$
u_{a}=\left(1+\tau_{a} s\right) R_{a} i_{a}+e_{m}
$$

where $u_{a}$ is the armature voltage; $i_{a}$ is the armature current; $\tau_{a}, R_{a}$ are the time's constant and the resistance of armature; $e_{m}$ is the motor's back-emf; $s=d / d t$ is the differentiation operator.

The equation of the motor's back-emf is given by:

$$
e_{m}=k_{\omega} \omega_{m}=k_{\omega} \dot{\varphi}_{m}
$$

where $\omega_{m}=\dot{\varphi}_{m}=s \varphi_{m}$-is the angular velocity of rotation of the shaft of motor; $k_{\omega}$ is the motor's back-emf constant.

The equation of the amplifier of power of the drive is:

$$
(1+\tau s) u_{a}=k u
$$

where $k, \tau$ are the coefficient of amplification and the time's constant of the amplifier of power; $u$ is the input voltage of the amplifier of power of the drive.

The equation of the moment of dc motor is:

$$
M_{m}=k_{m} i_{a}
$$

where $\mathrm{M}_{\mathrm{m}}$ is the moment of motor; $\mathrm{k}_{\mathrm{m}}$ is the motor's torque constant.

The "mechanical" part of the drive is described by the following equations [5]:

$$
\begin{gathered}
M_{m}=\left(J_{m}+J_{r}^{\prime}\right) \ddot{\varphi}_{m}+M_{L . m}+M_{R . m}, M_{L . m}=M_{L} / i, \\
M_{L}=J_{\varphi} \ddot{\varphi}+M_{\varphi}+M_{R . \varphi}, \ddot{\varphi}=\ddot{\varphi}_{m} / i,
\end{gathered}
$$

where $J_{m}+J_{r}^{\prime}$ is the sum of moments of inertia of motor's rotor and reducer specified to the shaft of motor; $M_{L . m}$ is the moment of load specified to the shaft of motor; $M_{R . m}$ is the resistance moments in motor and in reducer specified to the shaft of motor; $M_{L}$ - the resistance moment of load; $i$ is the gear ratio of reducer; $J_{\varphi}=J_{\varphi}\left(r, m_{p}\right)$ and $M_{\varphi}=$
$M_{\varphi}\left(r, \varphi, m_{p}\right)$ are the moment of inertia and the moment defined by Eq. (3); $M_{R . \varphi}$ is the resistance moment in joint of MR; $\ddot{\varphi}_{m}=\dot{\omega}_{m}=s \omega_{m}$-is the angular acceleration of rotation of shaft of motor.

Considering in common Eq. (8) neglecting the resistance moments $M_{R . m}, M_{R . \varphi}$, i.e. $M_{R . m}=$ $M_{R . \varphi}=0$, the "mechanical" part of drive is possible to describe as follows:

$$
J s \omega_{m}=M_{m}-M
$$

where $J=J_{m}+J_{r}^{\prime}+J_{\varphi} / i^{2}$ is the total moment of inertia of load counted to the shaft of drive motor; $M=M_{\varphi} / i$ is the resistance moment (external moment) of load counted to the shaft of drive motor.

Thus in Eq. (9) $J$ and $M$ variables depend on $J_{\varphi}$ and $M_{\varphi}$ quantities defined by Eq. (3) and characterize the variable mechanical load of the drive of a link of MR.

Solving in common Eqs. (4)-(7) and (9) taking into account the kinematic ratio $\omega=\omega_{m} / i=\dot{\varphi}_{m} / i$ and neglecting the lag effect of the electric circuit $\tau, \tau_{a}$, i.e. $\tau=\tau_{a}=0$, we will receive the following equation of the drive (of the plant) [5]:

$$
\frac{R_{a}}{k_{\omega} k_{m}} J s \omega+\omega=\frac{k}{i k_{\omega}} u-\frac{R_{a}}{i^{2} k_{\omega} k_{m}} M
$$

where $u$ is the control (command) signal of the plant.

The block diagram of the dc drive, i.e. of the plant, made on Eqs. (4)-(7) and (9) at $M_{R . m}=M_{R . \varphi}=0$ and $\tau=\tau_{a}=0$, is submitted on the upper right part of Fig. 1.

\section{Creation of a Load Adaptive Control System of Link Drive of Manipulator Robot}

In Eq. (10) the changes of $J$ and $M$ quantities will worsen dynamic properties of the SAC of the drive since $J$ is the changing (unknown) parameter and $M$ is the external disturbing signal for the plant.

Therefore for compensation of influence of variables of load to dynamical properties of the SAC of drive, we choose a control algorithm of drive, being adaptive to changes of the moment of inertia $J$ and the external moment $M$ of load of the drive as $[8,9]$ : 


$$
\begin{gathered}
u=u\left(\varphi, \omega_{m}, \hat{J}, \widehat{M}\right)=\left(k_{p} k_{1}\left(\varphi^{*}-\varphi\right)\right. \\
\left.-k_{s} k_{2} \omega_{m}\right) \hat{J}+k_{s} k_{3} \omega_{m}+k_{4} \widehat{M}
\end{gathered}
$$

where $\varphi^{*}, \varphi$ are respectively the demand and the actual angular positions of an output shaft of the drive; $k_{p}, k_{s}$ are respectively the transfer coefficients of sensors of angular position of a shaft of the drive and angular speed of a shaft of the motor; $k_{3}=k_{\omega} /\left(k_{s} k\right)$, $k_{4}=R_{a} /\left(k_{m} k\right)$ are the constant parameters; $\hat{j}$ and $\widehat{M}$ are the estimations of $J$ and $M$ quantities of the load received in the OD of identification of these quantities.

In Eq. (11) the $k_{1}, k_{2}$ constant parameters are selected with the accounting of values of $k_{m}, k_{\omega}, R_{a}$, $k, k_{p}$ and $k_{s}$ parameters so, that at $J=J_{0}=$ const and $M=0$, set in models of the drive (10), the desirable transition process of the SAC at $\varphi^{*}(t)=$ $1(t)$ will have the set duration $T, s$ and the set overshoot $\sigma, \%$ [9]:

$$
k_{1}=9 i R_{a} /\left(k k_{m} k_{p} T^{2}\right), k_{2}=6 R_{a} /\left(k k_{m} k_{s} T\right) .
$$

In our case for clear proof the desirable transition process is chosen aperiodic (monotonous) (Fig. 2, curve 3). Setting various values of the moment of inertia $J$ and the external moment $M$ of load in model of the drive (10) with the not adaptive to a load control algorithm when in Eq. (11) $\hat{J}=J_{0}=$ const and $\widehat{M}=0$ it is possible to see that to increase in the values of $J$ and $M$ there is deterioration in characteristics of transitional functions $h(t)=\varphi(t)$ (duration, overshoot and steady-state error increase)-in Fig. 2, curves 1 and 2. In this regard there is a problem of stabilization of desirable dynamic properties of the SAC of the drive with the variable mechanical load. The solution of this task is carried out due to development of the self-adjusted control system of the drive, adaptive to changes of the moment of inertia $J$ and the external moment $M$ of load which uncertain values are estimated in the identification OD which also is adaptive to changes of the specified quantities of drive's load.

In Refs. [10, 11] based on the main provisions of the theory of observing devices $[6,12]$ the algorithm of work of OD of identification of unknown value of moment of inertia of load of dc electric motor is proposed in following form:

$$
\begin{gathered}
d\left(k_{m} / \hat{J}\right) / d t=\delta_{1} i_{a} k_{s}\left(\omega_{m}-\widehat{\omega}_{m}\right) \\
d \widehat{\omega}_{m} / d t=\left(k_{m} / \hat{J}\right) i_{a}+\lambda_{1} k_{s}\left(\omega_{m}-\widehat{\omega}_{m}\right)
\end{gathered}
$$

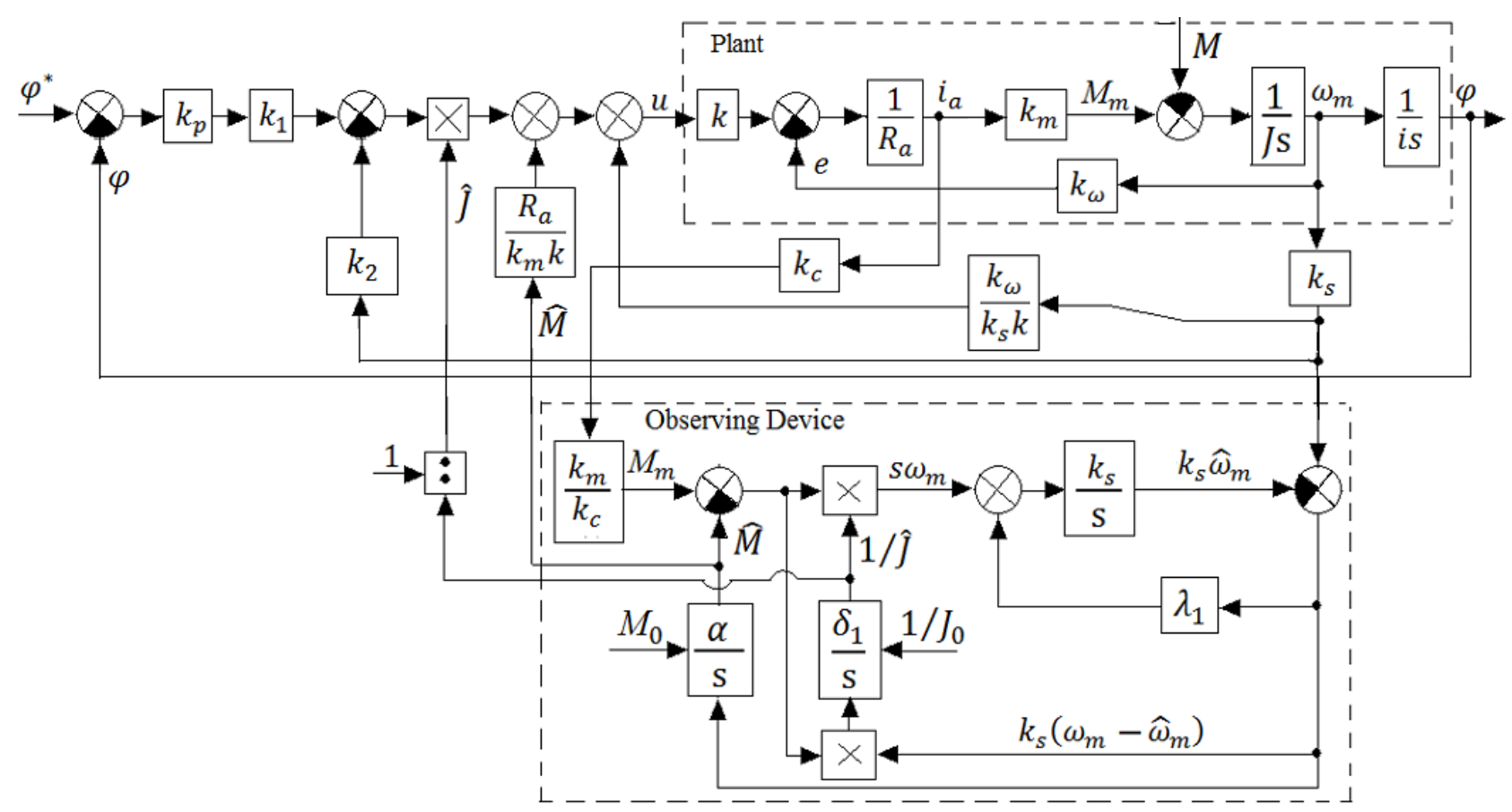

Fig. 1 Block diagram of the load adaptive system of automatic control of drive. 


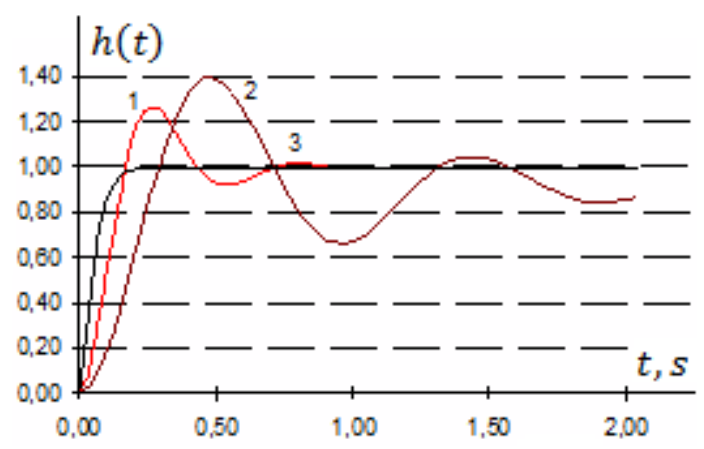

Fig. 2 The graphs of $h(t)$ transition functions. 1 -SAC without OD at $J=7 \mathrm{~kg} \cdot \mathrm{m}^{2}$ and $M=10 \mathrm{~N} \cdot \mathrm{m}$; 2-SAC without OD at $J=25 \mathrm{~kg} \cdot \mathrm{m}^{2}$ and $M=100 \mathrm{~N} \cdot \mathrm{m}$; 3-SAC with OD at the same values of $J$ and $M$ of load.

where $\hat{J}$ is the estimation of value of moment of inertia $J$ identified in the OD; $\widehat{\omega}_{m}$ is the estimation of the velocity of rotation of the shaft of motor $\omega_{m}$ received in the OD; $k_{s}$ is the transfer coefficient of sensor of angular velocity of a shaft of the motor; $\delta_{1}$, $\lambda_{1}$ are the constant coefficients.

However in this OD described by Eq. (12) the influence of the external moment $M$ of load of the motor is not considered, while it can cause the instability work of OD. Therefore in Ref. [13] by analogy with a "mechanical" part (9) of the DC motor the algorithm of work of OD of identification of moment of inertia of load is proposed in following form:

$$
\begin{gathered}
d(1 / \hat{J}) / d t=\delta_{1}\left(k_{m} i_{a}-M\right) k_{s}\left(\omega_{m}-\widehat{\omega}_{m}\right), \\
d \widehat{\omega}_{m} / d t=(1 / \hat{J})\left(k_{m} i_{a}-M\right) \\
+\lambda_{1} k_{s}\left(\omega_{m}-\widehat{\omega}_{m}\right)
\end{gathered}
$$

In Eq. (13) it is supposed that the external moment $M$ of load of the motor is measured by the sensor of moment and its value is known. However a measurement of the external moment $M$ is difficult to realize and it is not always possible, therefore it is required to develop the OD in which both the moment of inertia $J$ and the external moment $M$ of load are identified in common.

For obtaining of $\hat{J}, \widehat{M}$ estimations, the algorithm of operation of the OD of identification of the moment of inertia $J$ and the external moment $M$ of load has the following form [8]:

$$
\begin{gathered}
d(1 / \hat{J}) / d t=\delta_{1}\left(M_{m}-\widehat{M}\right) k_{s}\left(\omega_{m}-\widehat{\omega}_{m}\right), \\
d \widehat{\omega}_{m} / d t=(1 / \hat{J})\left(M_{m}-\widehat{M}\right)+\lambda_{1} k_{s}\left(\omega_{m}-\widehat{\omega}_{m}\right), \\
d \widehat{M} / d t=-\alpha k_{s}\left(\omega_{m}-\widehat{\omega}_{m}\right)
\end{gathered}
$$

with the initial conditions:

$$
\widehat{\omega}_{m}(0)=0, \hat{J}^{-1}(0)=J_{0}^{-1}, \widehat{M}(0)=0
$$

where $J_{0}$ is the average value from the possible range of changes of moment of inertia $J$.

In Eqs. (13) and (14) for formation of the moment of the motor $M_{m}$ according to Eq. (7) the armature current of motor $i_{a}$ which values are measured by the current sensor with transfer coefficient $k_{c}$ is used.

The block diagram of the load adaptive SAC of the drive consisting of a plant (10), control algorithm (11), OD of identification of variables of load (14) is shown in Fig. 1.

Let us consider the stability of the adaptive OD of identification of $J^{-1}$ and $M$ variables [14, 15]. Let us use the following designations:

$$
e=\omega_{m}-\widehat{\omega}_{m}, v=(1 / J)-(1 / \hat{J}), \mu=M-\widehat{M}
$$

and take into account that:

$$
s \omega_{m}=\dot{\omega}_{m}=d \omega_{m} / d t=(1 / J)\left(M_{m}-M\right)
$$

then the operation algorithm of the identification OD in $e, v$ and $\mu$ coordinates can be described by the equations:

$$
\begin{gathered}
d e / d t=(1 / J)\left(M_{m}-M\right) \\
-(1 / \hat{J})\left(M_{m}-\widehat{M}\right)-\lambda_{1} k_{s} e \\
d v / d t=-\delta_{1}\left(M_{m}-\widehat{M}\right) k_{s} e \\
d \mu / d t=\alpha k_{s} e
\end{gathered}
$$

At the same time we will accept initial conditions:

$$
e(0)=0, v(0)=J^{-1}-J_{0}^{-1}, \mu(0)=0,
$$

and on the basis of a hypothesis of quasi stationarity $[10,12]$ we will consider that on the time interval corresponding to transition process in OD the $J^{-1}$ and $M$ variables do not change.

Let us prove that the position of balance of system of Eq. (15) is asymptotically steady, i.e.

$$
\lim _{t \rightarrow \infty} e=0, \lim _{t \rightarrow \infty} v=0, \lim _{t \rightarrow \infty} \mu=0
$$

Let us consider a positive-definite function of Lyapunov of a following form:

$$
V=\frac{1}{2} e^{2}+\frac{1}{2 \delta_{1} k_{s}} v^{2}+\frac{1}{2 \alpha k_{s} J} \mu^{2}
$$


where $J=$ const - because it corresponds to a quasistationarity interval.

Considering that

$$
\mu=M-\widehat{M}=\left(M_{m}-\widehat{M}\right)-\left(M_{m}-M\right)
$$

Let us write down a full derivative of function $V$ with respect to time on the basis of system of Eq. (15):

$$
d V / d t=-\lambda_{1} k_{s} e^{2}
$$

Let us show that at $e \equiv 0$ there are also $v \equiv 0$ and $\mu \equiv 0$. For this purpose we will consider at $e \equiv 0$ the system of Eq. (15):

$$
\begin{gathered}
0=(1 / J)\left(M_{m}-M\right)-(1 / \hat{J})\left(M_{m}-\widehat{M}\right) \\
d v / d t=0, d \mu / d t=0
\end{gathered}
$$

The equality to zero of the first expression means $1 / J=1 / \hat{J}$ and $M=\widehat{M}$, therefore at $e \equiv 0$ the identical equality to zero the $v=(1 / J)-(1 / \hat{J})$ and $\mu=M-\widehat{M}$ parameters are obvious. Therefore the function $d V / d t$ is negative-definite and at construction the OD of identification according to Eq. (14) the $1 / \hat{J}$ and $\widehat{M}$ estimations asymptotically approach to their actual values of the moment of inertia $1 / J$ and the external moment $M$ of load of the drive. The convergence of process of estimate depends on $\lambda_{1}, \delta_{1}$ and $\alpha$ coefficients, which can be practically always chosen on a condition that the estimate processes in OD occur quicker than the main transition process in SAC of the drive.

\section{Results and Discussion}

Simulation of dynamics of control of the drive of rotation of a link of MR is carried out by method of numerical integration of Eq. (10) of the drive and writing the program on the computer. For this purpose Eq. (10) of plant with accounting of the kinematic relations of Eq. (8) was considered as the following system of differential equations [16, 17]:

$$
\begin{gathered}
d \omega_{m} / d t=(1 / J)\left(-k_{m} k_{\omega} R_{a}^{-1} \omega_{m}+k_{m} k R_{a}^{-1} u-M\right) \\
d \varphi / d t=\omega_{m} / i
\end{gathered}
$$

In the program of simulation the different values of the moment of inertia $J$ and the external moment $M$ of load are set in Eq. (10), these given values of load are estimated in identification OD on an algorithm (14) and used for formation of a control algorithm (11). Therefore, for simulation Eqs. (10), (11), (14) are necessary.

Results of simulation of dynamics of control of a rotation link drive of $\mathrm{MR}$ at variable values of mechanical load are given in Figs. 2-4. Modeling was carried out at the following parameters of the electric drive with DC motor and OD: $k=10 ; i=1 ; k_{p}=$ $\mathrm{V} / \mathrm{rad} ; k_{s}=3.5 \mathrm{Vs} / \mathrm{rad} ; k_{m}=0.7 \mathrm{~N} \cdot \mathrm{m} / \mathrm{A} ; k_{\omega}=0.8$ $\mathrm{V} \cdot \mathrm{s} / \mathrm{rad} ; R_{a}=3 \mathrm{Ohm} ; T=0.03 \mathrm{~s} ; \lambda_{1}=38 ; \delta_{1}=$ $0.0009 ; \alpha=3,000 ; J_{0}=16.5 \mathrm{~kg} \cdot \mathrm{m}^{2}$. So, in SAC without adaptation to load $\left(\hat{J}=\right.$ const $=J_{0}, \widehat{M}=0$ is not used) at working of an input signal $\varphi^{*}(t)=1(t)$ with increase in values of load happens the deterioration in characteristics of the transitional functions $h(t)$ of SAC (duration, overshoot and steady-state error increase)-in Fig. 2: curve 1 at $J=$ $7 \mathrm{~kg} \cdot \mathrm{m}^{2}$ and $M=10 \mathrm{~N} \cdot \mathrm{m}$; curve 2 at $J=25$ $\mathrm{kg} \cdot \mathrm{m}^{2}$ and $M=100 \mathrm{~N} \cdot \mathrm{m}$.

While in SAC with OD at these different values of mechanical load corresponding to different configurations of MR and masses of moved payloads, characteristics of transitional functions do not change (Fig. 2, curve 3), i.e. independent of changes of load in SAC with OD the set duration and overshoot of desired transition process always take place, and the steady-state error of the SAC is completely eliminated.

In Fig. 3, curves 1-4 of transition processes are shown in OD at the identification of unknown values of the moments of inertia $J$ set in the drive model (10) together with the external moments $M$ of load: 1 is the estimate of $J=7 \mathrm{~kg} \cdot \mathrm{m}^{2}$ at $M=10 \mathrm{~N} \cdot \mathrm{m} ; 2$ is the estimate of $J=10 \mathrm{~kg} \cdot \mathrm{m}^{2}$ at $M=40 \mathrm{~N} \cdot \mathrm{m}$; 3 is the estimate of $J=20 \mathrm{~kg} \cdot \mathrm{m}^{2}$ at $M=70 \mathrm{~N} \cdot \mathrm{m} ; 4$ is the estimate of $J=25 \mathrm{~kg} \cdot \mathrm{m}^{2}$ at $M=100 \mathrm{~N} \cdot \mathrm{m}$. In Fig. 4 the corresponding curves 1-4 are shown at the identification of unknown values of the external moment of load $M$ set in the drive model (10): 1 is the estimate of $M=10 \mathrm{~N} \cdot \mathrm{m}$ at $J=7 \mathrm{~kg} \cdot \mathrm{m}^{2} ; 2$ is the estimate of $M=40 \mathrm{~N} \cdot \mathrm{m}$ at $J=10 \mathrm{~kg} \cdot \mathrm{m}^{2}$; 3 is the 


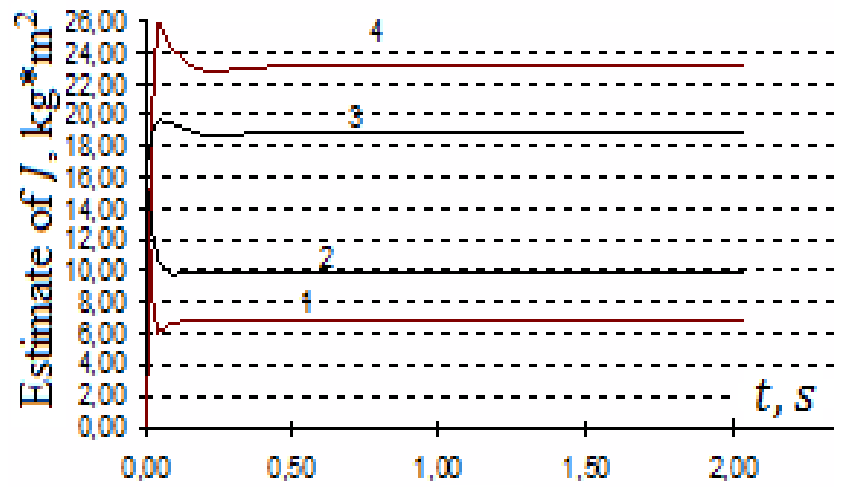

Fig. 3 Transition processes of identification of the moment of inertia of load. 1 is the estimate of $J=7 \mathrm{~kg} \cdot \mathrm{m}^{2}$ at $M=10 \mathrm{~N} \cdot \mathrm{m}$; 2 is the estimate of $J=10 \mathrm{~kg} \cdot \mathrm{m}^{2}$ at $M=40 \mathrm{~N} \cdot \mathrm{m}$; 3 is the estimate of $J=20 \mathrm{~kg} \cdot \mathrm{m}^{2}$ at $M=70 \mathrm{~N} \cdot \mathrm{m}$; 4 is the estimate of $J=25 \mathrm{~kg} \cdot \mathrm{m}^{2}$ at $M=100 \mathrm{~N} \cdot \mathrm{m}$.

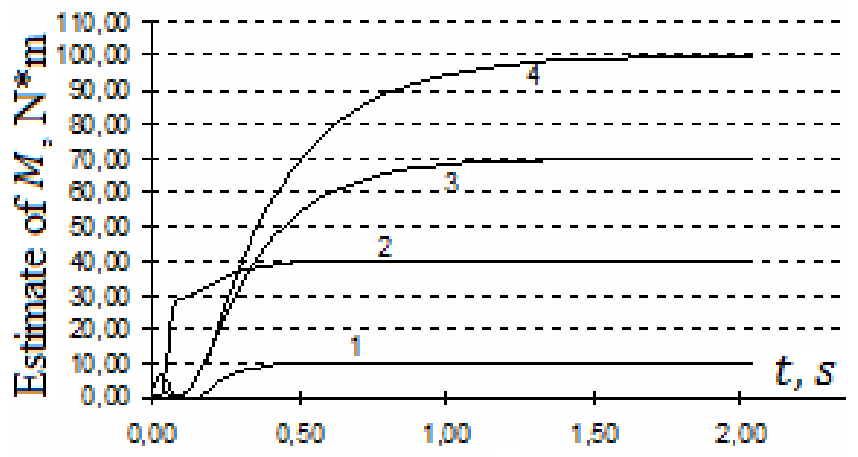

Fig. 4 Transition processes of identification of the external moment of load.

1 is the estimate of $M=10 \mathrm{~N} \cdot \mathrm{m}$ at $J=7 \mathrm{~kg} \cdot \mathrm{m}^{2}$;

2 is the estimate of $M=40 \mathrm{~N} \cdot \mathrm{m}$ at $J=10 \mathrm{~kg} \cdot \mathrm{m}^{2}$;

3 is the estimate of $J=20 \mathrm{~kg} \cdot \mathrm{m}^{2}$ at $M=70 \mathrm{~N} \cdot \mathrm{m}$;

4 is the estimate of $M=70 \mathrm{~N} \cdot \mathrm{m}$ at $J=25 \mathrm{~kg} \cdot \mathrm{m}^{2}$.

estimate of $J=20 \mathrm{~kg} \cdot \mathrm{m}^{2}$ at $M=70 \mathrm{~N} \cdot \mathrm{m} ; 4$ is the estimate of $M=70 \mathrm{~N} \cdot \mathrm{m}$ at $J=25 \mathrm{~kg} \cdot \mathrm{m}^{2}$.

From curves in Figs. 2-4 it is also visible that process of estimate of unknown variables of the moments of inertia $J$ happens quicker than transition process in the main contour of SAC, that is necessary for the steady work of a load adaptive SAC of the drive, and the estimated values of the unknown external moments $M$ of load eliminate a steady-state error of the drive.

The graphs in Figs. 2-4 of transition processes in OD at the identification of different values of the moment of inertia $J$ and the external moment $M$ of load set in model of the drive (10) have shown that the use of the values $J$ and $M$ estimated in OD to form an adaptive control algorithm (11) provides the invariable characteristics of transitional functions in the control system, i.e. independent of changes of mechanical drive load in SAC with OD the desirable transition process-curve 3 in Fig. 2 always takes place.

\section{Conclusion}

For an example of two-link polar MR is showed motions on coordinates of MR are interdependent and have caused the interference of coordinates at each other that will exert impact on control dynamics on separate coordinates. In dynamical Eq. (2) of rotary 
joint of MR, the moment of inertia of joint and the terms were allocated caused by inertial forces from interference of movements and gravity of links of MR. The moment of inertia of joint and the equation terms of interference and gravity of links are variables and together with the corresponding moment of inertia and the moment of mechanical resistance of the drive itself are the moment of inertia and the moment of resistance of variable mechanical load of the link drive of MR.

For the SAC of drive the moment of inertia of load is the parameter and the moment of resistance (external moment) of load is the external disturbing signal. Changes of these variables of load cause essential deterioration in dynamic properties (duration, overshoot and steady-state error increase) of the SAC. For the stabilization of desirable dynamic properties of the SAC of drive it is required to use the actual values of the moment of inertia and the external moment of load to form a control algorithm of the drive. However when the MR is moving unknown payloads the values of these parameters of load will be unknown. For the identification of the unknown parameters and external disturbing signals of the SAC it uses the ODs [6, 10-12]. So, in Refs. [10,11] the algorithm of work of OD of identification of unknown value of moment of inertia of load of DC electric drive is proposed. However in this OD described by Eq. (12) the external moment $M$ of drive load is not considered, while it can cause the instability work of OD. Therefore in Ref. [13] by analogy with a "mechanical” part (9) of the drive the algorithm (13) of OD of identification of moment of inertia of load of drive is proposed in which the external moment $M$ of drive load is considered. As in algorithm (13) the measurement of the external moment $M$ is difficult to realize and it is not always possible, in Ref. [8] the algorithm (14) of the OD was proposed in which both the moment of inertia $J$ and the external moment $M$ of load are identified in common.

The proposed algorithm (14) of OD has asymptotically stability of identification of unknown values of the moment of inertia and the external moment of load of the drive during necessary time that allows their use in an algorithm of adaptive control (11) and provides elimination of their disturbing influence on dynamics of control of the drive (10) of a link of MR.

Results of simulation on the computer illustrate that, first, variables of the unknown parameters of load of joint-drive of MR cause inadmissible deterioration in dynamic properties of the SAC of drive, and secondly, use in a load adaptive algorithm (11) of control of the drive (10) instead of unknown values of the moment of inertia $J$ and the external moment $M$ of load of the drive their values $\hat{J}$ and $\widehat{M}$ estimated in the OD described by Eq. (14) provides stabilization of desirable dynamic properties of the SAC of link drive of MR. The set of a load adaptive drive control systems each of which is in the degree of mobility of MR will provide desirable dynamics of control of movement of end-effector of MR in the course of performance of production tasks.

\section{References}

[1] Craig, J. J. 2005. Introduction to Robotics: Mechanics and Control. Upper Saddle River, NJ: Pearson Education, Inc.

[2] Lewis, F. L., Dawson, D. M., and Abdallah, C. T. 2004. Robot Manipulator Control: Theory and Practice. New York, Basel: Marcel Decker, Inc.

[3] Yurevich, E. I., ed. 1984. Dynamics of Control of Robots. Moscow: Science. (in Russ.)

[4] Krutko, P. D. 1991. Control of Actuating Systems of Robots. Moscow: Nauka. Gl. Red. Phis.-Mat. Lit. (in Russ.)

[5] Burdakov, S. F., Dyachenko, V. A., and Timofeev, A. N. 1986. Design of Manipulators of Industrial Robots and Robotic Complexes: Textbook for Students. Moscow: Vyssh. shkola. (in Russ.)

[6] Kuzovkov, N. T. 1976. Modal Control and the Observing Devices. Moscow: Mashinostrojenie. (in Russ.)

[7] Mohan, N. 2003. Electric Drives: An Integrative Approatch. Minneapolis: MNPERE.

[8] Dzholdasbekov, U. A., Moldabekov, M. M., and Kussainov B. K. 1999. "Adaptive control system of the drive” Preliminary Patent No. 7672 RK. Bulletin No. 6: 6. 
(in Russ.)

[9] Sholanov, K. S., and Kussainov, B. K. 2008. "Adaptive Control of the Robot Drive." Bulletin of National Engineering Academy of Sciences of RK 4: 75-81. (in Russ.)

[10] Basharin, A. V., Novikov, V. A., and Sokolovskij, G. G. 1982. Control of Electric Drives: Textbook for Students. Leningrad: Energoizdat. Leningradskoje otdelenije. (in Russ.)

[11] Selivanov, V. A. 2010. Control Systems of Electric Drives: Textbook for Students. Mogilev: Academy. (in Russ.)

[12] Alexandrov, A. G. 2003. Optimum and Adaptive Systems. Moscow: Vysshaya shkola. (in Russ.)

[13] Dzholdasbekov, U. A., Moldabekov, M. M., and Kussainov B. K. 1997. "Control System of the Robot's Drive.” Preliminary Patent No. 5616 RK. Bulletin No. 5:

\author{
6. (in Russ.)
}

[14] Kussainov, B. K. 2009. "Adaptive Control System of the Robot Drive.” In Proceedings of the International Forum "Science and Engineering Education without Borders" V.2. Almaty: KazNTU named after K. I. Satpayev, 352-55. (in Russ.)

[15] Sholanov, K. S., and Kussainov, B. K. 2010. "Adaptive Control of the Robot Drive." Bulletin of National Engineering Academy of Sciences of RK 3: 87-92. (in Russ.)

[16] Kussainov, B. 2018. “A Load Adaptive Control System of Manipulator Robot's Drive.” In Proceedings of IV International Scientific Congress "Innovations 2018”. Year II, 1 (2): 78-81.

[17] Kussainov, B. 2018. “A Load Adaptive Control System of Manipulator Robot's Drive.” International Scientific Journal “Innovations”. Year VI, 3: 78-81. 